\title{
PREPAREDNESS OF THE BHAKTI WIRATANTAMA ARMY HOSPITAL SEMARANG IN FACING COVID-19
}

\author{
Moh. Andi Fatkhurokhman'), Budi Hidayat²) \\ ${ }^{1)}$ Hospital Administration Studies, Faculty of Public Health, \\ Universitas Indonesia, Depok, West Java, Indonesia \\ ${ }^{2)}$ Health Policy and Administration, Faculty of Public Health, \\ Universitas of Indonesia, Depok, West Java, Indonesia
}

\begin{abstract}
Background: On March 11, 2020, World Health Organization (WHO) established Covid-19 as a pandemic. Coronavirus is one of the large family of viruses that cause illnesses ranging from mild symptoms such as fever, coughing and shortness of breath, some of the case without symptoms. Coronavirus severe symptoms such as pneumonia, acute respiratory syndrome, kidney failure, and even can cause death. Coronavirus is transmitted between animals and humans. The average incubation period is about 6 to14 days. This study aimed to describe the preparedness of the Bhakti Wira Tantama Army Hospital Semarang, Central Java in facing the Covid-19 outbreak.

Subjects and Method: A mix method study was conducted at Bhakti Wira Tantama Army Hospital Semarang, Central Java. The process of organizing data in this study began with the literature study of health regulatory and laws in Indonesia. The instrument of this study was based on the Hospital Readiness checklist from WHO guidelines. The other data were collected using in-depth interviews. The data then analyzed descriptively.

Results: Bhakti Wira Tantama Hospital was sufficient for a maximum capacity of 48 Covid19 patients. In details, it showed that communication (87.49\%), continuity of essential health services and patient care (100\%), surge capacity (57.02\%), human resources (56.40\%), logistic and management of supplies including pharmaceuticals (51.51\%), surveillance early warning and monitoring (100\%), essential support services (95.23\%), case management (59.88\%), infection prevention and control (58.96\%), and laboratory services (53.85\%).
\end{abstract}

Conclusion: Bhakti Wira Tantama Army Hospital already have a very good level of preparedness in facing the Covid-19 pandemic.

Keywords: Covid-19, hospital readiness, world health organization

\section{Correspondence:}

Moh. Andi Fatkhurokhman. Hospital Administration Studies, Faculty of Public Health University of Indonesia, Depok, West Java, Indonesia. Email: andi.sptht@gmail.com. Mobile: 082135806088 